\title{
The magnetic reversal characteristics of 32- bit composite element magnetic barcodes
}

Cite as: Appl. Phys. Lett. 115, 162404 (2019); https://doi.org/10.1063/1.5119075

Submitted: 08 July 2019. Accepted: 04 October 2019 . Published Online: 15 October 2019

P. J. Newton (D), L. De Los Santos Valladares (D, R. Celis Rojas, and C. H. W. Barnes (D)
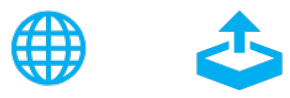

\section{ARTICLES YOU MAY BE INTERESTED IN}

Spintronic GdFe/Pt THz emitters

Applied Physics Letters 115, 152401 (2019); https://doi.org/10.1063/1.5120249

Temperature-dependent spin Hall effect tunneling spectroscopy in platinum

Applied Physics Letters 115, 162403 (2019); https://doi.org/10.1063/1.5121165

Van der Waals heterostructures of blue phosphorene and scandium-based MXenes monolayers

Journal of Applied Physics 126, 143101 (2019); https://doi.org/10.1063/1.5114850

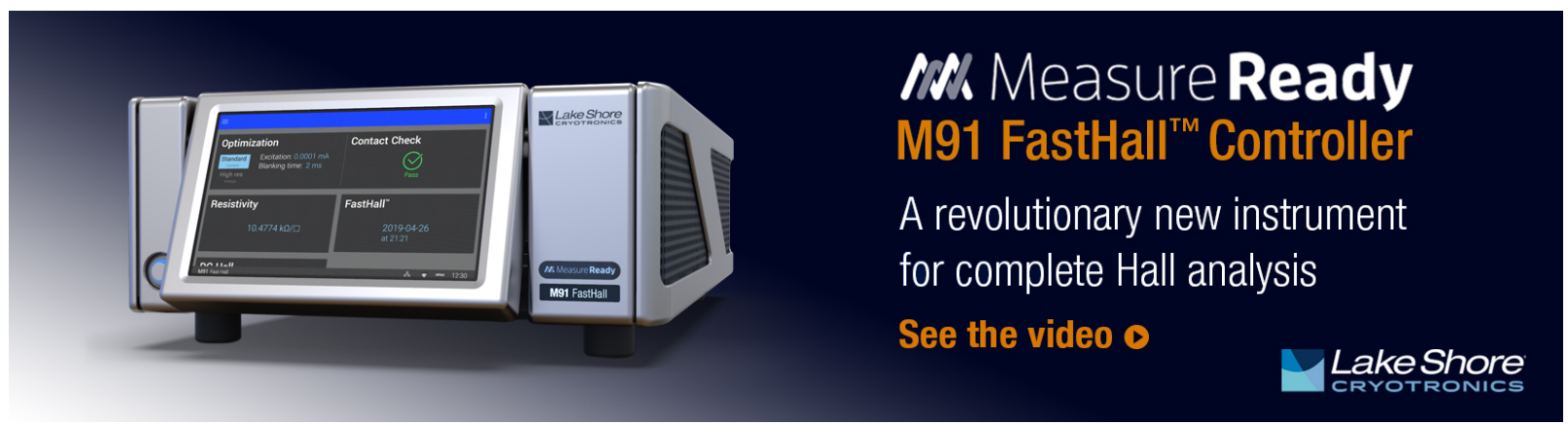




\title{
The magnetic reversal characteristics of 32-bit composite element magnetic barcodes
}

\author{
Cite as: Appl. Phys. Lett. 115, 162404 (2019); doi: 10.1063/1.5119075 \\ Submitted: 8 July 2019 - Accepted: 4 October 2019 . \\ Published Online: 15 October 2019
}



P. J. Newton, ${ }^{1, a)}$ (D) L. De Los Santos Valladares, ${ }^{1,2,3}$ (D) R. Celis Rojas, ${ }^{4,5}$ and C. H. W. Barnes ${ }^{7}$ (D)

\begin{abstract}
AFFILIATIONS
'Department of Physics, Cavendish Laboratory, University of Cambridge, J. J. Thomson Avenue, Cambridge CB3 OHE, United Kingdom

${ }^{2}$ School of Materials Science and Engineering, Northeastern University, No 11, Lane 3, Wenhua Road, Heping District, Shenyang 110819, People's Republic of China

${ }^{3}$ Laboratorio de Superconductividad, Facultad de Ciencias Físicas, Universidad Nacional Mayor de San Marcos, Ap. Postal 14-0149, Lima 15081, Peru

${ }^{4}$ Laboratorio de Celdas Solares, Universidad Nacional de Barranca, Av. Toribio de Luzuriaga N³76, Mz J, Urbanización La Florida, Distrito y Provincia de Barranca 15169, Peru

${ }^{5}$ Laboratorio de Ciencias, Universidad Nacional Autónoma de Tayacaja “Daniel Hernandez Morillo," Jr., Bolognesi 418, Pampas 09156, Huancavelica, Peru
\end{abstract}

a) Author to whom correspondence should be addressed: pjn32@cam.ac.uk

\begin{abstract}
Magnetic barcodes containing 32 composite element bits have been produced and measured in order to optimize the design of magnetic microcarriers. Focused magneto-optic Kerr effect measurements allow the determination of the change in magnetic hysteresis when the width of magnetic elements is varied between bits, and the electron beam lithography used in production is confirmed to be accurate to $\sim 6 \mathrm{~nm}$ using scanning electron microscopy. The sharp magnetic switching observed, an important prerequisite for a functioning device, is attributed to the expected dipolar interactions between magnetic elements and the use of magnetically soft Permalloy. A crossover between two magnetic reversal behaviors is discovered when the magnetic elements are $\sim 200 \mathrm{~nm}$ wide. From these measurements, 12 bits were selected on which data can be written with a low probability of error, with the prospect of the other 20 bits being employed for error correction. We have therefore developed a nonvolatile magnetic memory on which 4096 unique codes can be programmed.
\end{abstract}

Published under license by AIP Publishing. https://doi.org/10.1063/1.5119075

Composite element magnetic barcodes are versatile, nonvolatile magnetic memories that exploit dipolar interactions between, ${ }^{1}$ and the shape anisotropy of, ${ }^{2}$ narrow magnetic strips to allow a binary code to be written under the application of an appropriate global magnetic field. ${ }^{3-7}$ These barcodes make magnetically encoded microcarriers relevant for rapid and noninvasive detection, identification, and sorting of compounds in biomolecular libraries, e.g., in microfluidic split-andmix processes. ${ }^{8}$

Batches of microcarriers have been produced with surfaces functionalized with two different probe molecules on their top and bottom faces, respectively. ${ }^{9,10}$ By functionalizing the surface of the tags with an appropriate receptor, with a quantifiable fluorescence when in the presence of a target heavy metal anion, this scheme could find applications in water quality testing. When the barcodes fluoresce in this way, the stray fields of the microcarriers can be measured, in flow, using a tunneling magnetoresistance (TMR) sensor, ${ }^{11}$ to identify the heavy metal that is measured. From this, it should be possible to obtain a quantifiable measure of a heavy metal concentration.

Recent work has focused on the development of the composite element bit design, where the magnetic elements are composed of parallel, rectangular strips with very large aspect ratios that are coupled by dipolar interactions. ${ }^{1}$ This design overcomes the limitations of using barcodes where bits are simply composed of individual, high aspect ratio rectangles: individual, small elements cannot be detected at large distances in flow and switching occurs over a large magnetic field range, resulting in the unintentional switching of many bits when attempting to write onto the barcode. ${ }^{3,4}$

The ability to design bits such that they exhibit a sharp magnetic switching at more equally spaced magnetic fields will allow for an optimal ease of programming. However, previous composite element barcode studies have been limited to the investigation of only a small number of possible bit designs, many of which have very similar 
switching fields. ${ }^{1}$ This makes extrapolation of designs for working devices difficult. We have made numerous unsuccessful attempts to fabricate barcodes containing bits with equally spaced coercivities by treating coercivity as having a single power law dependence on the strip width. An analysis of devices containing many more bits is vital, therefore, either as a functional device in its own right or as a basis for future designs. In this work, we investigate 32 possible magnetic bits with strip widths in the range of 100-3200 nm. By analyzing the magnetic properties of these bits, we are able to suggest 12 bits on which data could be reliably written with a small probability of error.

Figure 1(a) shows a schematic of a typical 5-bit tag design. The device here is illustrated as being encapsulated in SU8. The SU8 layer was left out of the devices in this work in order to maximize signal-tonoise ratios during magnetic measurements. Figure 1(b) shows a magnified image of the design for bit 2. This also defines the terms used in the description of the bits and barcodes. Individual bits are separated by comparatively large distances, ensuring that magnetostatic interactions between bits are vanishingly small. ${ }^{12}$ Bits therefore have only a very weak influence on the switching of neighboring bits in this design. ${ }^{13}$

Throughout this work, magnetically soft Permalloy $\left(\mathrm{Ni}_{80} \mathrm{Fe}_{20}\right)$ is used as the ferromagnetic material in the bits. Previous work has shown that this material gives a sharp magnetic switching upon application of an appropriate magnetic field - a feature that is essential for the operation of magnetic barcodes. ${ }^{1}$ All magnetic elements have a length of $15 \mu \mathrm{m}$-sufficiently long to ensure that magnetic behavior is dominated by shape anisotropy and dipole-dipole interactions and that there is a good signal-tonoise ratio in magneto-optic Kerr effect (MOKE) measurements.

To produce the devices measured in this work, electron-beam patterning of a single layer of $950 \mathrm{~K}$ polymethyl-methacrylate (PMMA) A4 was performed on a Si substrate coated with a capped $\mathrm{Al}$ release layer to mimic the conditions required for production of magnetic microcarriers. These samples were then developed in a 5:15:1 ratio of methylisobutyl-ketone:isopropanol:methyl-ethyl-ketone (MIBK:IPA:MEK). A thin $\mathrm{Cr}$ adhesion layer, $\mathrm{Ni}_{80} \mathrm{Fe}_{20}(15 \mathrm{~nm})$, and a $\mathrm{Au}(10 \mathrm{~nm})$ capping layer were grown by thermal evaporation in an Edwards Auto 306 evaporator at a base pressure of less than $3 \times 10^{-7}$ mbar. Finally, liftoff was performed in acetone in order to form the separated magnetic bits on the surface.

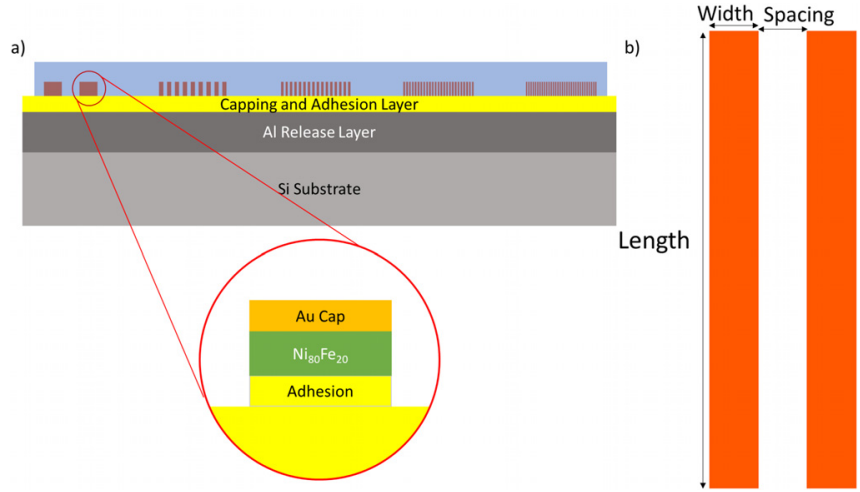

FIG. 1. A schematic diagram (not to scale) of the magnetic barcode design. (a) A complete 5-bit barcode design, shown encapsulated in SU8. The layer structure of the magnetic element is also shown. (b) An individual bit (bit 2 in this case) illustrating the terms used in descriptions.
In order to characterize the accuracy of the electron beam lithography used to define the magnetic strips, scanning electron microscopy (SEM) images were obtained. These data, made using an FEI Phillips XL30 FEG ESEM and a $5 \mathrm{kV}$ accelerating voltage, are displayed in Fig. 2. An SEM image of a single device is shown in Fig. 2(a), along with a more magnified image of a portion of bit 32 (with designed strip widths and spacing of $100 \mathrm{~nm}$ ) in Fig. 2(b). The bright regions correspond to the strips themselves (containing the magnetic material), and the darker regions are the troughs between them. Here, the accuracy of the lithography can be clearly seen. An average discrepancy of only about $6 \mathrm{~nm}$ is observed between the designed and measured strip widths, with the width of the strips in bit 32 being $94 \pm 7 \mathrm{~nm}$, with this standard deviation largely attributable to edge roughness of the strips. This is a clear indication of the precise control of strip dimensions, which is essential to obtain the desired magnetic properties of the bits.

Hysteresis loops were obtained by measuring the longitudinal magneto-optic Kerr effect (MOKE) using a nanoMOKE 2 magnetometer, produced by Durham Magneto Optics Ltd. A scanning MOKE image of a 32-bit device was used to locate individual bits, alongside reference to live optical images. The laser spot was focused to a fullwidth-half-maximum diameter of $5 \mu \mathrm{m}$. Very precise alignment of the laser spot to the center of each bit was required to obtain symmetric hysteresis loops. The MOKE data used for this analysis are the result of normalizing the average of 50 loops measured in quick succession on a single bit.

Sharp switching behavior is seen in the hysteresis of the bits. From these MOKE data, the coercivity is extracted using the Level Crossing tool on Origin. From the over 1000 barcodes produced in this $5 \mathrm{~mm} \times 5 \mathrm{~mm}$ total area, five were chosen at random and all 32 bits measured. Figure 3 shows the mean coercivity of each bit against its width as blue diamonds, along with data points for each individual measurement as pink stars. The error bars show the standard deviation of the coercivity at each strip width. Looking at this plot, there appear to be two distinct regions with different functional dependences on the coercivity. Power law fits to these regions are shown in Fig. 3(b) with functions of $H_{C}=A_{1} w^{-1.32}$ for widths $100-152 \mathrm{~nm}$ and $H_{C}=A_{2} w^{-0.82}$ for widths $200-3200 \mathrm{~nm}$, where $A_{1}$ and $A_{2}$ are the fitting prefactors. The points between are ignored for the purposes of fitting, since they are best modeled as being a crossover of the two regimes explored below. These results are consistent with our previous



FIG. 2. SEM images of a 32-bit device showing (a) the entirety of a whole device and (b) a highly magnified image of an area of bit 32 , where strip widths are designed to equal $100 \mathrm{~nm}$. 


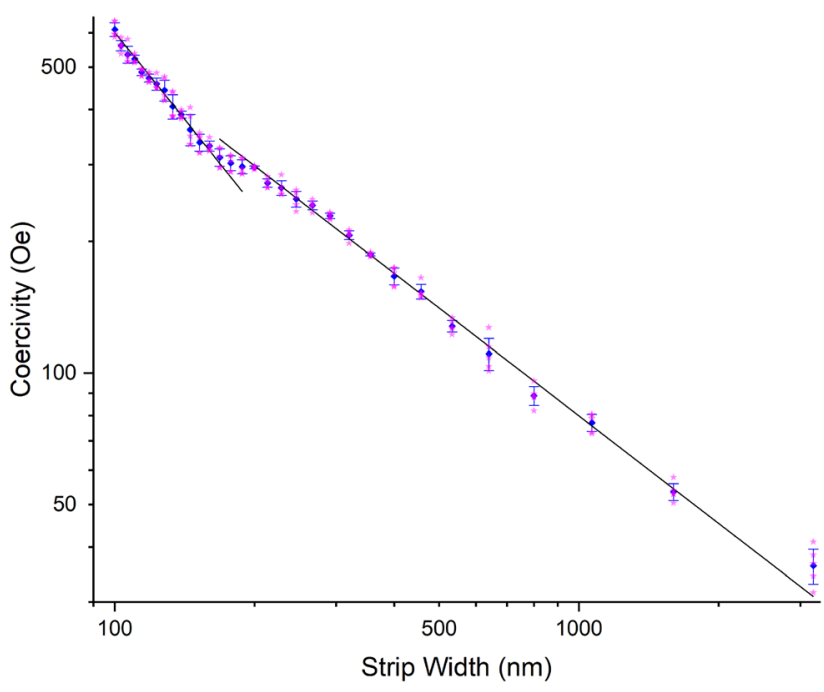

FIG. 3. The variation of magnetic coercivity of composite element bits with a change in the strip width. Blue diamonds are averages of all measurements, and error bars display the standard deviation of the coercivity for each width. Trendlines shown are power law fits to these data.

unsuccessful attempts to construct barcodes containing bits with equally spaced coercivities by treating this relationship as a single power law.

Being a thermally evaporated film, there is no lattice anisotropy associated with a crystal lattice. The magnetic easy axis is entirely determined by the shape anisotropy created during lithography. The difference in the power law relationship of magnetic coercivity to the strip width can be explained by a change in the switching mechanism, and remnant magnetization, as strips get wider.

The narrowest strips undergo a single domain switching-the shape anisotropy here preventing multidomain structures from forming. ${ }^{14}$ In this mechanism, the exact field at which individual strips switch is quite dependent on their particular individual shape, and variation in the shape along their length [as can be seen in the SEM image of Fig. 2(b)] and at their corners, although these structures are sufficiently narrow that the coercivity should be independent of edge roughness from lift-off. ${ }^{15,16}$ The single domain switching is thought to nucleate at defects, which will again clearly vary from strip to strip.

In contrast, wider strips can support multiple domains at remanence. Specifically, there will be partial flux closure domains at the corners of wider magnetic strips. ${ }^{17}$ Being wider, there is also less impact of edge roughness and the small variations in shape along the length of the strip. This, alongside the presence of dipolar interactions between strips, ensures a very sharp switching behavior.

Previous measurements on a number of polycrystalline materials have shown that there should be a reciprocal relationship between the width and magnetic coercivity of a single, multidomain magnetic element. ${ }^{18-20}$ There are a number of possible explanations for the exponent of -0.82 measured in this work. Dipolar interactions are known to be have more influence over wider magnetic elements. ${ }^{13}$ This leads to an enhancement of coercivity for wider magnetic strips, as stray fields from neighboring elements result in an effectively smaller local field being experienced and hence a reduction in the power-law exponent. In addition, edge roughness is expected to enhance the coercivity of wide magnetic rectangles but have little impact on those with widths as small as $200 \mathrm{~nm} \cdot{ }^{15,18}$ Again, this would effectively enhance the coercivity of wider strips and reduce the power-law exponent.

With careful consideration of the need to incorporate equal amounts of magnetic material in each bit, it should be possible to reach even larger coercivities by modifying the shapes of the ends of each magnetic strip. Having a pointed end with a length of twice the width of each strip could double the switching field, allowing the exploration of a much larger parameter space. ${ }^{21}$

This device could not be used to store 32-bit codes in its current form. Some of the bits appear to be functionally identical in MOKE measurements-c.f. bits 16, 17, and 18 in Fig. 3. It should, however, be possible to use this 32-bit barcode to store shorter binary codes by selecting an appropriate routine. For example, Fig. 4 gives the parameters for a scheme where 12 bits are used to store up to $2^{12}=4096$ unique binary codes. A table showing the parameters involved in this potential scheme is shown in Fig. 4(a), along with the magnetic properties of these 12 bits in Fig. 4(b), here showing the magnetic hysteresis of the element with median coercivity for each bit.

There is no overlap in the standard deviation of the coercivity of these 12 bits, meaning that only a small number of barcodes would need to be read out, in flow, in a potential assay, to have a high a)

\begin{tabular}{|c|c|}
$\begin{array}{c}\text { Bit } \\
\text { Number }\end{array}$ & $\begin{array}{c}\text { Average Measured } \\
\text { Coercivity (Oe) }\end{array}$ \\
\hline $\mathbf{I}$ & $36 \pm 3$ \\
\hline $\mathbf{2}$ & $54 \pm 2$ \\
\hline 3 & $77 \pm 3$ \\
\hline $\mathbf{5}$ & $110 \pm 9$ \\
\hline $\mathbf{8}$ & $166 \pm 7$ \\
\hline 10 & $207 \pm 5$ \\
\hline 13 & $250 \pm 10$ \\
\hline 20 & $331 \pm 9$ \\
\hline 23 & $389 \pm 7$ \\
\hline 26 & $458 \pm 14$ \\
\hline 29 & $522 \pm 11$ \\
\hline 32 & $610 \pm 23$ \\
\hline
\end{tabular}

b)

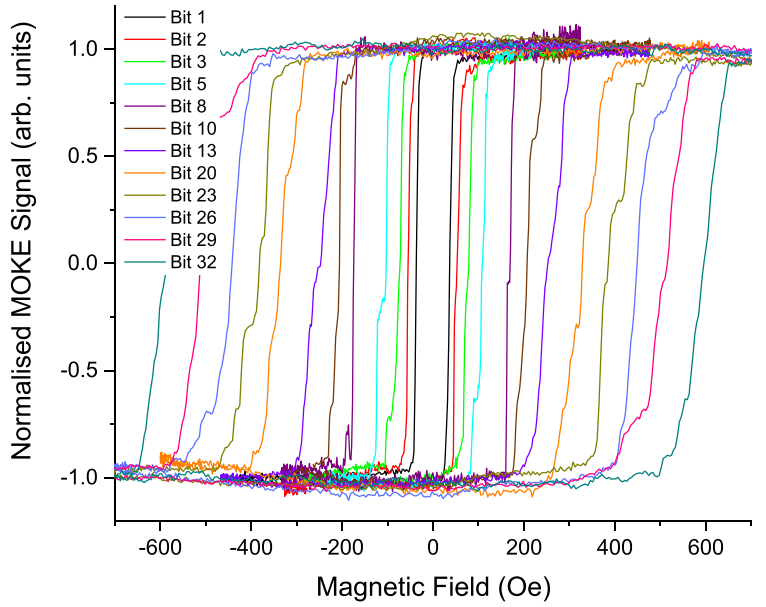

FIG. 4. (a) The average coercivity of the bits to be programmed in the 12-bit scheme. Errors are the standard deviations in measurements of the coercivity of each bit. (b) The magnetic hysteresis of each magnetic element with the median coercivity of the sample set. 
confidence of the code that was written onto the barcode. There is, however, some overlap in the switching of the bits with the narrower strip widths. There is less influence of dipolar interactions on narrower magnetic elements. ${ }^{13}$ This results in each element in a bit switching at a slightly different field, dependent on its exact width, and giving the broad switching that is observed. This can be seen quite clearly in Fig. 4(b) with the presence of shoulders in the hysteresis of bit 23 , for example. In a barcode device, this broad switching could result in these bits not being fully magnetized after a code is programmed and a smaller signal upon readout. Further development into devices with a smaller strip spacing would mitigate these effects by increasing the dipolar interaction between narrow strips and promote a sharper switching behavior.

Additionally, neighboring bits to the ones primarily used to store the sequence could be used as error correction for individual bit flips or variations in properties of an individual barcode that may have led to incorrect writing of a bit. Alternatively, increasing the number of barcodes used in an individual assay could lead to a corresponding increase in the number of bits that could be utilized to store data. The utility of the presence of error correction bits is compensated for by the increased averaging of barcodes.

Codes can be written to these barcodes by the application of appropriate magnetic field sweeps. For example, to program code 000000000011 , the device is initially saturated to the 0 state with a 700 Oe in-plane field. A sweep back to -65 Oe reverses the magnetization state of only bits 1 and 2 and results in the code 000000000011 . The writing of this code can be verified by magnetic force microscopy measurements. More complicated codes can be built up in a similar way using alternating field sweeps (for instance, 000000001100 is written with sweeps of 700 Oe to -138 Oe to 65 Oe). These fields must be very precisely obtained during the sweep to minimize the partial magnetic reversal of neighboring bits, particularly when considering the bits with the narrowest widths.

In conclusion, the production of a 32-bit barcode and its measurement using MOKE has allowed the development of a scheme to write a 12-bit binary sequence, with other bits being used for error correction. The coercivity of composite element magnetic bits is found to be a binary, power-law function of strip width. The crossover between the two regimes at $\sim 200 \mathrm{~nm}$ is attributed to a change in magnetic reversal behavior.

See the supplementary material for magnetic force microscopy measurements of a device after encoding.

This work was carried out initially with the financial support of the Universidad Nacional de Moquegua, Peru, and completed with the help of the Universidad Nacional de Barranca, Peru. The authors would particularly like to acknowledge Alberto Quispe
Cohaila (from No. RG85120) and Luis Enrique Carrillo Diaz (from No. RG92973) for their encouragement and constant support during each project. We must also thank Eustace P. G. Barnes, D. H. Nimalika Perera, and Noorhayati Idros for helpful discussions on the field applications for these tags in water analysis.

\section{REFERENCES}

'D. M. Love, K. N. Vyas, A. Fernandez-Pacheco, J. Llandro, J. J. Palfreyman, T. Mitrelias, and C. H. W. Barnes, RSC Adv. 5, 10211 (2015).

${ }^{2}$ A. O. Adeyeye, J. A. C. Bland, C. Daboo, J. Lee, U. Ebels, and H. Ahmed, J. Appl. Phys. 79, 6120 (1996).

${ }^{3}$ J. R. Jeong, J. Llandro, B. Hong, T. J. Hayward, T. Mitrelias, K. P. Kopper, T. Tryiniotis, S. J. Steinmuller, G. K. Simpson, and J. A. C. Bland, Lab Chip 8, 1883-1887 (2008).

${ }^{4}$ B. Hong, T. J. Hayward, J. R. Jeong, J. F. K. Cooper, J. J. Palfreyman, T. Mitrelias, A. Ionescu, J. A. C. Bland, and C. H. W. Barnes, J. Appl. Phys. 105, 034701 (2009).

${ }^{5}$ T. Mitrelias, J. F. K. Cooper, K. N. Vyas, J. J. Palreyman, B. Hong, T. J. Hayward, and C. H. W. Barnes, J. Appl. Phys. 107, 09B319 (2010).

${ }^{6}$ T. J. Hayward, B. Hong, K. N. Vyas, J. J. Palfreyman, J. F. K. Cooper, Z. Jiang, J. R. Jeong, J. Llandro, T. Mitrelias, J. A. C. Bland, and C. H. W. Barnes, J. Phys. D: Appl. Phys. 43, 175001 (2010).

${ }^{7}$ J. J. Palfreyman, P. Beldon, B. Hong, K. N. Vyas, J. F. K. Cooper, T. Mitrelias, and C. H. W. Barnes, 8th International Conference on the Scientific and Clinical Applications of Magnetic Carriers 99 (2010), pp. 184-191.

${ }^{8}$ J. Llandro, J. J. Palreyman, A. Ionsecu, and C. H. W. Barnes, Med. Biol. Eng. Comput. 48, 977 (2010).

${ }^{9}$ K. N. Vyas, J. J. Palreyman, D. M. Love, T. Mitrelias, and C. H. W. Barnes, Lab Chip 12, 5272-5278 (2012).

${ }^{10}$ J. Palfreyman, D. Love, A. Philpott, K. Vyas, C. Cimorra, T. Mitrelias, C. Barnes, L. Muir, G. Cook, and R. Keynes, IEEE Trans. Magn. 49, 285-295 (2013).

${ }^{11}$ K. N. Vyas, B. Hong, J. F. Cooper, J. J. Palfreyman, and C. H. W. Barnes, IEEE Trans. Magn. 47, 1571-1574 (2011).

${ }^{12}$ E. Y. Tsymbal, Appl. Phys. Lett. 77, 2740 (2000).

${ }^{13}$ K. Szulc, F. Lisiecki, A. Makarov, M. Zelent, P. Kuświk, H. Głowiński, J. W. Kłos, M. Münzenberg, R. Gieniusz, J. Dubowik, F. Stobiecki, and M. Krawczyk, Phys. Rev. B 99, 064412 (2019).

${ }^{14}$ R. P. Cowburn, J. Phys. D: Appl. Phys. 33, R1 (2000).

${ }^{15}$ M. T. Bryan, D. Atkinson, and R. P. Cowburn, Appl. Phys. Lett. 85, 3510 (2004).

${ }^{16}$ S. H. Liou, R. F. Sabiryanov, S. S. Jaswal, J. C. Wu, and Y. D. Yao, J. Magn. Magn. Mater. 226-230, 1270-1272 (2001).

${ }^{17}$ K. J. Kirk, J. N. Chapman, and C. D. W. Wilkinson, Appl. Phys. Lett. 71, 539 (1997).

${ }^{18}$ W. C. Uhlig and J. Shi, Appl. Phys. Lett. 84, 759 (2004).

${ }^{19}$ A. Fernández-Pacheco, J. M. De Teresa, A. Szkudlarek, R. Córdoba, M. R. Ibarra, D. Petit, L. O’Brien, H. T. Zeng, E. R. Lewis, and D. E. Read, Nanotechnology 20, 475704 (2009).

${ }^{20}$ R. D. Shull, Yu. P. Kabanov, V. S. Gornakov, P. J. Chen, and V. I. Nikitenko, J. Magn. Magn. Mater. 400, 191-199 (2016).

${ }^{21}$ K. J. Kirk, J. N. Chapman, and C. D. W. Wilkinson, J. Appl. Phys. 85, 5237 (1999). 